\title{
Expectation and autokinesis
}

\author{
H. W. LEIBOWITZ, C. L. SHUPERT, R. B. POST, and J. DICHGANS \\ Pennsylvania State University, University Park, Pennsylvania
}

\begin{abstract}
The reported direction of autokinesis for naive observers is not biased by gaze deviations of $22.5 \mathrm{deg}$ of arc. However, instructions to expect either "inward" or "outward" movement produce reports of autokinetic movement that are progressively biased in the expected direction. Instructions to expect "more" or "less" movement resulted in a decrease in the reported duration of apparent movement for the "less" group but no change for the "more" group. Response bias is not considered to play a role in these results. The results are discussed in terms of fixational bias and selective attention mechanisms of autokinesis.
\end{abstract}

It has been recognized since the classical studies of Sherif (1935) that suggestion and expectation play a role in the reported magnitude and direction of autokinesis (AK). Indeed, the sensitivity of AK to social pressure and personality factors has resulted in an extensive literature (see Levy, 1972, and Royce, Carran, Aftanas, Lehman, \& Blumenthal, 1966, for reviews). However, AK is also of interest from the point of view of sensory mechanisms, in particular the oculomotor system. In such studies it is important that the conditions of testing preclude the possibility that suggestion and expectation could influence the results.

We recently analyzed the contribution of gaze deviation in AK, the literature of which dates back almost a century (Charpentier, 1886; Holmgren, 1889). It was our intention to reexamine this phenomenon in the light of recent developments in our understanding of gaze stability mechanisms (Leibowitz, Shupert, Post, \& Dichgans, 1983; Post, Shupert, \& Leibowitz, 1983). Many previous AK studies, particularly those involving social factors, requested that the subject numerically estimate the magnitude of AK movement. Since such numerical estimates are not verifiable with respect to a physical scale, it is perhaps not surprising that social factors are effective in biasing AK responses.

In the initial phase of our studies, we attempted to devise a methodological technique that we expected would be insensitive to expectations or suggestions. A physical reference was provided by activating a

This research was supported by Grants MH08061 from the National Institutes of Health and EY02376 from the National Eye Institute, and by a Senior Scientist Award (to H.W.L.) from the Alexander von Humboldt Foundation for study at the Department of Neurology, University of Freiburg. The authors are grateful to Muzafer Sherif for his encouragement. Current addresses are: R. B. Post, Department of Ophthalmology, University of California, Davis, California 95616; J. Dichgans, Department of Neurology, University of Tübingen, Tubingen, West Germany. Reprint requests should be addressed to: $H$. Leibowitz, Moore Building, Pennsylvania State University, University Park, Pennsylvania 16802 . metronome at 1 tick/sec during AK observation. The subject was asked to indicate the direction of $\mathbf{A K}$ movement and, using the metronome as a reference, to estimate the duration of such movements. We had assumed that the reported duration and direction of AK would be less sensitive to suggestion. This assumption was consistent with the research of Wallace, Garrett, and Anstadt (1974), who failed to find an effect of suggestion on the reported direction of AK. Using this technique, we proceeded to investigate the variable in which we were interested, the role of gaze deviation.

The initial studies proceeded satisfactorily. The method was readily applicable, and the preliminary data appeared to indicate that, as expected, gaze deviation biases AK. The original data were obtained using the four authors as subjects. Even though the direction of AK was reversed for one of us, we were confident that eccentric fixation was a major contributing factor to the direction of AK movement and proceeded to prepare a manuscript for publication. Fortunately, we also decided to test additional, naive subjects. While the preparation of the manuscript proceeded smoothly, confirmatory evidence from the additional subjects was not obtained for the moderate eccentricities (22.5 deg) employed in our study. Naive subjects did not produce response patterns related to gaze deviation until they were made aware of the hypothesis under consideration. The present study reports data from initially naive subjects for whom expectancies were systematically introduced.

\section{EXPERIMENT 1}

\section{Method}

Six males and 9 females who ranged in age from 19 to 30 years and who were naive to the purpose of the experiment were assigned randomly to one of three groups of five subjects each. They were tested individually in three sessions, with at least $24 \mathrm{~h}$ intervening between sessions. At the beginning of each session, the subject was seated at a chinrest $50 \mathrm{~cm}$ in front of a screen that filled the entire field of view. The nondominant eye was patched, and the subject was instructed to fixate a point on a photoflash that was masked so 
as to generate a rectangular afterimage subtending $4.5 \times 2.25 \mathrm{deg}$, with an unstimulated circular area $2.25 \mathrm{deg}$ in diameter in the center. After the flash had been activated, the room lights were extinguished and the subject allowed the afterimage to fade. When fading was complete, the subject illuminated the screen with a flashlight and marked the position of the renewed afterimage. Approximately seven such positions were determined. The median of the horizontal and vertical distributions of the marks was used as the subject's resting direction. Following the determination of the resting direction, the subject was asked to observe the $\mathbf{A K}$ movement of a light-emitting diode ( $8 \mathrm{~min}$ of arc at a $50-\mathrm{cm}$ viewing distance; luminance $=.8 \mathrm{~cd} / \mathrm{m}^{2}$ ) affixed to the screen at one of five positions: at the subject's resting direction, and at 22.5 deg directly up, down, right, or left of the resting direction. Three 20 sec trials were carried out for each position of the diode, and positions were presented in random order. On each trial, the subject rested for $20 \mathrm{sec}$ with eyes closed, after which the experimenter gave a verbal command to start observation. The subject then observed the light-emitting diode for $20 \mathrm{sec}$ while listening to a metronome ticking at $1 \mathrm{~Hz}$ and then reported the number of seconds of AK movement observed in each of eight directions: up, down, left, right, and the four obliques. At the conclusion of the Day 1 test session, one group of five subjects was told that, although at first most subjects see random $\mathbf{A K}$ movement at all positions of the diode, with practice, all subjects begin to see the AK stimulus move away from the resting direction (outward) at all eccentric positions. These subjects were then shown their data sheets, and any data conforming to the expected pattern were pointed out to them. The same procedure was carried out at the conclusion of Day 2 and at the beginning of Day 3. A second group of five subjects was treated similarly, except that they were told that all subjects tend to see movement toward the resting direction (inward) at all eccentric positions. The third group of five subjects, which was given no information, served as a control.

\section{Results}

The data, presented as the percentage of time directional AK movement was reported, are described in Figure 1. For all subjects for Day 1 (left column), and also for the noninformed control group on Days 2 and 3 (middle row), the reports of apparent movement are largely unrelated to the direction of gaze deviation. It should be noted that most of the movement, as is typically observed, is in the up-down direction, but is not systematically related to the direction of gaze deviation. The middle and right columns of the top row present data for those subjects for whom the "out" bias expectations were given on Day 2. Their reports demonstrate a tendency for outward movement on Day 2 and an even greater tendency on Day 3. On Day 3,65\% of the movement is in the expected direction.

For the "in" expectation group (bottom row), a similar influence of expectation is observed. There is a tendency toward inward motion, that is, motion opposite to gaze deviation, on Day 2, and this tendency increases on Day 3. Eighty-five percent of the reports on Day 3 were in the expected direction.

A statistical analysis of the data supports these conclusions. The number of seconds of north, south, east, or west movement reported by each subject was summed across the three trials and then analyzed in a $3 \times 3 \times 5 \times 4$ (instruction group $\times$ day $\times$ stimulus position $\times$ direction of movement) analysis of vari-

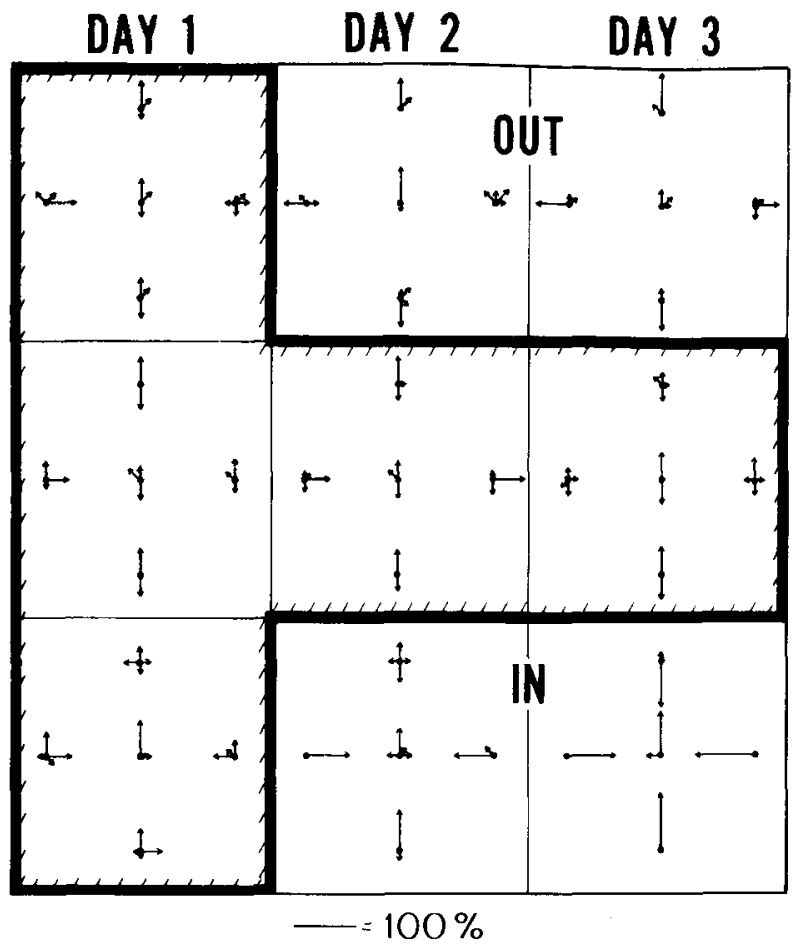

Figure 1. The effect of suggestion on the directional bias of autokinetic (AK) movement. Data were obtained for a stimulus at the resting direction (central diagram of each square) and for gaze deviations of $22.5 \mathrm{deg}$ in the four cardinal directions. The length of the vector is proportional to the percentage of time directionally blased AK movement was observed. No suggestions were given on Day 1. On Days 2 and 3, "out" suggestions were given to the group whose data are indicated in the top row, and "in" suggestions to the group whose data are presented in the bottom row. For the other group (middle row), no bias directions were given.

ance with repeated measures on the last three factors. The only significant main effect was direction of movement $[F(3,36)=4.91,36, p<.01]$, reflecting the tendency for north movement to be reported significantly more often than other directions. A significant interaction was reported between stimulus position and direction of movement $[F(12,144)=5.96$, $p<.011$, reflecting the variation in north movement across stimulus positions: more north movement is reported for north, south, and resting direction positions than for east and west positions. Finally, significant interactions were found among stimulus position, direction of movement, and group $[F(24,144)=3.33, p<.01]$, and among days, stimulus position, direction and group $[F(48,288)=$ $1.50, \mathrm{p}<.01]$, indicating a significant effect of instructions on the reported direction of AK movement.

Clearly, the direction of reported AK is systematically related to the observer's expectations. For the subjects who were not given expectations, gaze deviations of $22.5 \mathrm{deg}$ did not result in a systematic bias of AK direction. However, when it was suggested that AK is related to gaze deviation, the subjects' direc- 
tional responses were progressively biased in the expected direction.

Although we were aware that $\mathbf{A K}$ is highly susceptible to suggestion and expectations (Crutchfield \& Edwards, 1949; Sherif, 1935), we were surprised by these results. The classical studies by Sherif (1935) on suggestion and AK utilized, for the most part, the response measure of magnitude of reported movement. We had reasoned that direction of movement, as used in this study, would be less susceptible to expectations. Furthermore, since all of the author-observers in the first phase of this study were experienced in psychophysical observations, we assumed that we were not susceptible to expectations, particularly when making directional judgments. Obviously, both of our assumptions were incorrect. Expectation is a major factor in AK. These data have clear and profound methodological implications.

Since many investigations have been concerned with stimulus parameters that tend to increase or decrease the amount of illusory movement reported, the degree to which these reports can be affected by subjects' expectations is also significant. In view of the strong effect of demand characteristics (Orne, 1970) on the reported direction of AK movements, a second experiment was performed to determine whether the duration of AK movement could also be influenced by suggestion.

\section{EXPERIMENT 2}

\section{Method}

Six male and nine female volunteers participated in the study. The subjects, who were recruited from elementary psychology courses and were naive to the purpose of the experiment, were divided randomly into three groups. They were tested individually in three sessions, with at least $24 \mathrm{~h}$ intervening between sessions. At the beginning of each session, the subject was seated in a chair and provided with a headrest. All observations were made monocularly with the dominant eye. Each session consisted of two 180 sec trials, in which subjects were asked to observe a light-emitting diode affixed to a screen straight ahead at eye level, and to indicate when the diode appeared to move by depressing a switch connected to a timer. At the end of the first session, one group of five subjects was told that, with practice, all subjects see more illusory movement during the allotted time. The subjects in this group were then shown their data sheets, and data conforming to this pattern were pointed out to them. The same procedure was carried out at the conclusion of Day 2 and at the beginning of Day 3. A second group was treated similarly, except that they were told that all subjects tend to see less illusory movement with practice. The third group of subjects, which was given no information, served as a control.

\section{Results}

The results of Experiment 2 are presented in Figure 2, which shows the mean percentage of time that AK was reported on each trial for each group. A $3 \times 3 \times 2$ (instruction group $\times$ day $\times$ trial) analysis of variance with repeated measures for sessions and trials revealed no significant main effects, but a sig-

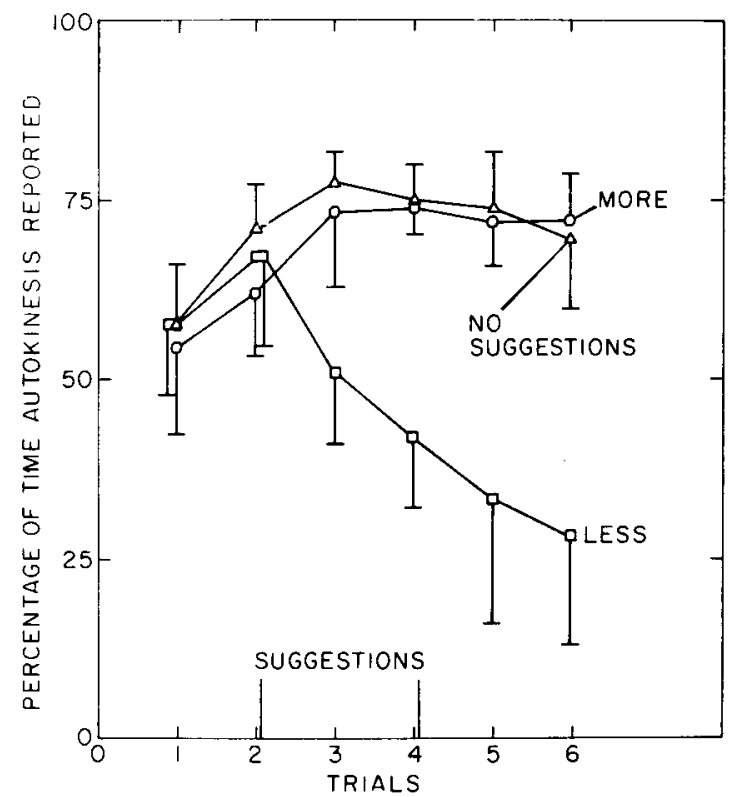

Figure 2. The effect of suggestion on the durntion of reported autokinetic movement. On Day 1 (Trials 1 and 2), no suggestions were given. Instructions to see "more" or "less" movement (or no instructions) were given at the end of Days 1 and 2 (after Trials 2 and 4 ). Error bars represent one standard deviation.

nificant session $\times$ groups interaction $[F(4,24)=2.99$, $\mathrm{p}<.05]$ was found. A Newmann-Keuls procedure indicated that the mean for the third session for the "less" expectation group was significantly different from the means for all other groups in all sessions. It should be noted that the results from the "more" expectation group did not differ from the results of the control group, which indicated that expectation alone is not sufficient to increase the duration of $\mathbf{A K}$ movement reported.

\section{DISCUSSION}

The principal finding of this study is that expectations, even for experienced psychophysical observers, play a major role in the direction of reported AK. Such results are consistent with the previous literature on the effects of suggestion. They also raise a question as to the mechanism(s) by which expectation can influence the direction of $\mathbf{A K}$. We would like to suggest some possibilities:

Fixational bias-During attempted fixation, retinal image movement is readily appreciated by the fovea because of its high sensitivity to displacement, and it constitutes a stimulus for voluntary eye movement in the interest of preventing loss of fixation. Since voluntary effort involves activation of the pursuit system, an uncanceled efference copy (von Holst \& Mittelstaedt, 1950) that results in ap- 
parent movement is produced. In the absence of a systematic bias from the vestibular or oculomotor systems, the direction of these efforts and the accompanying illusory motion sensations would be random. However, if the observer expects motion in a specific direction, it is possible that the subsequent fixation efforts would be modified so that AK progressively corresponds to the anticipated direction of illusory motion. Because the awareness of the effort associated with the initiation of pursuit movements is generally low or absent, this voluntary bias of the effort to maintain fixation could be initiated without the observer's awareness. Additionally, the ability of subjects to influence fixational drift could also result in a bias of the direction of retinal image motion.

Selective attention-Since the eyes are not perfectly stable during fixation of a stationary stimulus, involuntary eye movements would be likely to stimulate different directionally specific motion analyzers. Additionally, it is assumed that even in the absence of retinal image motion, motion analyzers sensitive to different directions of motion have a resting or tonic level of activity (Barlow \& Hill, 1963). If subjects are expecting motion in a particular direction, it is possible that they could selectively attend to the activity of particular subsets of motion detectors (Ball \& Sekuler, 1980), with the consequence that motion in these directions is perceived.

The results of Experiment 2 indicate that expectation can decrease, but not increase, the reported duration of AK, which suggests that neither the effects on duration nor those on direction are due simply to response bias. The findings of Wallace et al. (1974), who failed to report an effect of suggestion on the duration of $\mathrm{AK}$, are also consistent with this notion. In Wallace et al.'s study, the suggestion was delivered only once prior to a single testing session; if suggestion effects are due merely to response bias, a single suggestion should have been sufficient to produce the effect. In our studies, significant changes in duration and direction were obtained only after repeated suggestions. This finding implies that some sort of "learning" may be involved in suggestion effects. The ability of subjects to decrease the amount of AK they report has interesting implications for the role of eye movements in AK. Subjects may be able to learn to suppress eye movements during attempted fixation. This possibility is consistent with the results of Kowler and Steinman
(1979), who reported that some subjects are able to reduce the number of microsaccades normally exhibited during attempted fixation.

We have no basis for selecting among these alternatives or for not considering the possibility that some combination of mechanisms is involved. The conclusion that expectation apparently has sensory consequences (as opposed to response bias) has clear and profound methodological implications. Whatever the mechanism of the influence of expectation on $\mathbf{A K}$, it is clear that unbiased results in studies of AK can be obtained only from subjects who have no expectations regarding this phenomenon.

\section{REFERENCES}

Ball, K., \& Seruler, R. Models of stimulus uncertainty in motion perception. Psychological Review, 1980, 87, 435-470.

Barlow, H. B., \& Hilt, R. M. Evidence for a physiological explanation of the waterfall phenomenon and figural aftereffects. Nature, 1963, 200, 1345-1347.

Charpentier, A. Sur une illusion visuelle. Comptes Rendues Academie des Sciences, Paris, 1886, 102, 1155-1157.

Crutchfield, R., \& EDwards, $W$. The effect of a fixated figure on autokinetic movement. Journal of Experimental Psychology, $1949,39,561-568$.

Holmgren, F. Studien über die elementaren Farbenempfindungen. Skadinavisches Archiv für Physiologie, 1889, 1, 152-183.

Kowlen, E., \& Steinman, R. M. Miniature saccades: Eye movements that do not count. Vision Research, 1979, 19, 105-108.

Leibowitz, H. W., Shupert, C. L., Post, R. B., \& Dichgans, J. Autokinetic drifts and gaze deviation. Perception \& Psychophysics, 1983, 33, 455-482.

LEVY, J. Autokinetic illusion: A systematic review of theories, measures, and independent variables. Psychological Bulletin, $1972,78,457-474$.

Orne, M. T. Hypnosis, motivation, and the ecological validity of the psychology experiment. In W. J. Arnold \& M. M. Page, Nebraska Symposium on Motivation (Vol. 18). Lincoln: University of Nebraska Press, 1970.

Post, R. B., Shupert, C. L., \& Leibowitz, H. W. Autokinesis and peripheral stimuli. Perception, 1983, 11, 477-482.

Royce, J. R., Carran, A. B., Aftanas, M., Lehman, R. B., \& Blumenthal, A. The autokinetic phenomenon: A critical review. Psychological Bulletin, 1966, 65, 243-260.

Shenif, M. A study of some social factors in perception. Archives of Psychology, 1935, 187, $60 \mathrm{pp}$.

von Holst, E., \& MitTelstaedt, H. Das Reafferenzprinzip: Wechselwirkungen zwischen Zentralnervensystem und Peripherie. Naturwissenschaften, 1950, 37, 464-476.

Wallace, B., Garrett, J., \& Anstadt, S. Hynotic susceptibility, suggestion, and reports of autokinetic movement. American Journal of Psychology, 1974, 87, 117-123.

(Manuscript received September 21, 1981; revision accepted for publication February 24, 1983.) 